\title{
Daptomycin for Gram-positive Infections in Patients with Neutropenia: Clinical Experience from a European Outcomes Registry
}

Felix Keil · George L. Daikos · Athanasios Skoutelis · Jose Ignacio Barranco Dominguez •

Rashidkhan Pathan · Kamal Hamed (D)

To view enhanced content go to www.advancesintherapy.com

Received: June 23, 2015 / Published online: August 4, 2015

(C) The Author(s) 2015. This article is published with open access at Springerlink.com

\section{ABSTRACT}

Introduction: The aim of this analysis was to describe in real-world settings the clinical outcomes and safety associated with daptomycin treatment in patients with neutropenia and Gram-positive infections.

Electronic supplementary material The online version of this article (doi:10.1007/s12325-015-0231-3) contains supplementary material, which is available to authorized users.

F. Keil

Hanusch Krankenhaus der Wiener

Gebietskrankenkasse-Medizinische Abteilung,

Heinrich Collin-Strasse, Vienna, Austria

G. L. Daikos

National University of Athens-Laiko General

Hospital, Athens, Greece

A. Skoutelis

Evangelismos General Hospital, Athens, Greece

J. I. B. Dominguez

Hospital Universitario Miguel Servet, Zaragoza, Spain

R. Pathan

Novartis Healthcare Pvt. Ltd, Hyderabad, India

K. Hamed ( $\square)$

Novartis Pharmaceuticals Corporation,

East Hanover, NJ, USA

e-mail: kamal.hamed@novartis.com
Methods: Patients with an absolute neutrophil count (ANC) $\leq 1000$ cells $/ \mathrm{mm}^{3}$ who received at least one dose of daptomycin between 2006 and 2012 were selected from a non-interventional, multicenter, retrospective registry (European Cubicin $^{\circledR}$ Outcome Registry and Experience; EU-CORE ${ }^{\mathrm{SM}}$ ).

Results: Of the 6075 patients enrolled in EU-CORE, 446 (7.3\%) had an ANC s 1000 cells $/ \mathrm{mm}^{3}$ at baseline or during daptomycin therapy; they were all included in efficacy and safety populations. Half of the patients had severe neutropenia (ANC $\leq 100$ cells $/ \mathrm{mm}^{3}$ ). Most patients had hematologic malignancy (60.5\%), an immunosuppressed state $(39.7 \%)$ or had undergone a transplant (27.8\%). The most common primary infections were bacteremia (42.2\%) and complicated skin and soft tissue infection (13.9\%). Cultures were positive for $68.6 \%(254 / 370)$ of patients with available culture results; coagulase-negative staphylococci (43.7\%; 111/254) and Staphylococcus aureus $(18.9 \% ; 48 / 254)$ were the most commonly isolated primary pathogens. Median duration of daptomycin therapy was 10.0 (range 1-98) days. Most patients (82.8\%) received antibiotics concomitantly with 
daptomycin; the most common were carbapenems $(51.2 \%)$, penicillins $(42.1 \%)$, and aminoglycosides (19.9\%). The overall clinical success rate (cured or improved) associated with daptomycin was $77.1 \%$. Adverse events possibly related to daptomycin treatment were reported in seven (1.6\%) patients and led to drug discontinuation in 27 (6.1\%) patients.

Conclusion: The study results suggest that daptomycin is an effective therapeutic option for the treatment of a broad range of Gram-positive infections in patients with neutropenia, and has a good safety profile.

Funding: This study was funded by Novartis Pharma AG.

Keywords: Daptomycin;

Gram-positive infections; Neutropenia;

Staphylococci

\section{INTRODUCTION}

Patients with profound and prolonged neutropenia are at a major risk of infection [1]. Febrile neutropenia is generally a complication of myelosuppressive chemotherapy and requires immediate evaluation to avoid progression to a sepsis syndrome and possibly death [2, 3].

Gram-positive bacteria are currently the leading agents responsible for infections in patients with neutropenia worldwide [4]. Moreover, a major concern is that resistant Gram-positive pathogens, such as methicillin-resistant Staphylococcus aureus (MRSA) and vancomycin-resistant enterococci (VRE), have become common in these patients. Thus, in some centers, MRSA and VRE are the most prevalent resistant isolates, accounting for up to $20 \%$ and $50 \%$ of episodes, respectively [5].

In patients with fever and neutropenia, recent guidelines do not recommend empiric therapy with antimicrobials, such as vancomycin, linezolid or daptomycin, but suggest early administration of these agents for patients with specific clinical indications or who are at risk of exposure to resistant bacteria [5]. These situations include patients with hemodynamic instability, suspected catheter-related infection, skin or soft tissue infection, pneumonia, previous infection or colonization with MRSA or VRE, or a hospital setting with high rates of resistant Gram-positive pathogens [5].

Vancomycin has been the mainstay of therapy for infections caused by resistant Gram-positive pathogens, such as MRSA, for several decades [6]. However, in patients with febrile neutropenia, it is associated with a delayed response and the development of resistant organisms [7]. Moreover, recent guidelines recommend using alternatives when the vancomycin minimum inhibitory concentration (MIC) of the infecting MRSA strain is increased $[8,9]$. Indeed, many reports suggest a link between a higher vancomycin MIC of the infecting pathogen and a worse clinical outcome in patients with an MRSA infection [10].

To improve the outcome of these vulnerable patients with neutropenia, daptomycin is an attractive agent because of its broad spectrum of activity and its bactericidal action. It is a lipopeptide antibiotic with rapid bactericidal activity against most clinically relevant Gram-positive pathogens, including many antibiotic-resistant strains [11-13]. Daptomycin has not been formally evaluated in randomized trials in patients with neutropenia, but several case reports and abstracts have been published documenting its potential in this setting [14-16]. The retrospective analysis from the Cubicin ${ }^{\circledR}$ Outcome Registry and Experience (CORE) 
showed that daptomycin was effective and well tolerated in patients with neutropenia [17].

The European CORE (EU-CORE ${ }^{\mathrm{SM}}$ ) was designed to collect real-world demographic and clinical data on patients treated with daptomycin. The goal of the present analysis was to describe the clinical outcome and safety of daptomycin in patients with neutropenia treated for documented Gram-positive infections.

\section{METHODS}

\section{Study Design and Data Collection}

EU-CORE was a non-interventional, multicenter, retrospective, patient registry designed to collect real-world outcome in patients treated with daptomycin for Gram-positive infections. Detailed EU-CORE methodology has been described elsewhere [18]. Briefly, local investigators from 18 countries in Europe (12), Latin America (5), and Asia (1) collected demographic, primary infection, prior and concomitant antibiotics, clinical, and microbiologic data using standardized case-report forms for patients with Gram-positive infections who had received at least one dose of daptomycin between January 2006 and April 2012, and had had at least 30 days of post-treatment follow-up. Patients who had received daptomycin as part of a controlled clinical trial were excluded.

For the present analysis, patients with an absolute neutrophil count (ANC) $\leq 1000$ cells/ $\mathrm{mm}^{3}$ at baseline or during daptomycin treatment were selected. Patients were stratified into three categories based on the ANC: those with severe $\left(\leq 100\right.$ cells $\left./ \mathrm{mm}^{3}\right)$, moderate (101-499 cells $\left./ \mathrm{mm}^{3}\right)$, and mild (500-1000 cells $\left./ \mathrm{mm}^{3}\right)$ neutropenia $[5,19]$.
Institutional review board (IRB) approval was obtained before the start of the study and all procedures followed were in accordance with the ethical standards of the responsible committee on human experimentation (institutional and national) and with the Helsinki Declaration of 1964, as revised in 2013, and Good Clinical Practices. Informed consent was obtained according to the requirements of the IRB and/or local data privacy regulations.

\section{Clinical Outcomes and Safety}

Local investigators assessed clinical outcomes at the end of daptomycin therapy according to protocol-defined criteria: (1) cured, clinical signs and symptoms resolved, no additional antibiotic therapy was necessary, or infection cleared with a negative culture reported; (2) improved, partial resolution of clinical signs and symptoms and/or additional antibiotic therapy was warranted; (3) failed, inadequate response to daptomycin therapy, worsening or new/recurrent signs and symptoms, need for a change in antibiotic therapy, or positive culture reported at the end of the therapy; and (4) non-evaluable, unable to determine response because of insufficient information [20]. Clinical success was used to describe collectively patients with an outcome of cured or improved. Time to improvement was also recorded. Duration of treatment was measured as the number of inpatient and outpatient days during which the patient received daptomycin therapy, even if these were non-consecutive.

Adverse events (AEs) and serious AEs (SAEs) during daptomycin treatment, and 30-day follow-up period were assessed by the investigators. All deaths, AEs, and SAEs were recorded, regardless of their relation to daptomycin. 


\section{Statistical Analysis}

The safety population included all eligible patients with at least one safety assessment and the efficacy population included all eligible patients for whom clinical outcome was assessed.

Given that this was a registry, no inferential analyses were conducted and no formal statistical methodology other than simple descriptive statistics was used. All analyses were considered to be explanatory. Continuous variables were summarized as arithmetic mean, standard deviation, median, and range; categorical variables were summarized by absolute and relative frequencies.

Statistical analysis was performed using SAS version 9.3 (SAS Institute Inc., Cary, NC, USA).

\section{RESULTS}

\section{Patient Demographic and Clinical Characteristics}

Of the 6075 patients enrolled in EU-CORE registry who had received at least one dose of daptomycin, 446 (7.3\%) had an ANC $\leq 1000$ cells $/ \mathrm{mm}^{3}$ at baseline or during daptomycin treatment; 50\% $(n=223)$ had severe neutropenia (ANC $\leq 100$ cells $/ \mathrm{mm}^{3}$ ). All patients selected for the present analysis were included in both safety and efficacy populations. Baseline demographics and clinical characteristics of patients are summarized in Table 1. Overall, $58.5 \%(n=261)$ of patients were men and $26.2 \%(n=117)$ had an age $\geq 65$ years. The most common underlying diseases were hematologic malignancy $(60.5 \%$; $n=270)$, immunosuppressed state $(39.7 \%$; $n=177)$, and transplant $(27.8 \% ; n=124)$.
Table 1 Demographic and clinical characteristics

\begin{tabular}{|c|c|}
\hline Characteristics & $\begin{array}{l}N=446 \\
n(\%)\end{array}$ \\
\hline Male gender & $261(58.5)$ \\
\hline \multicolumn{2}{|l|}{ Age, years } \\
\hline Median (range) & $54.5(4-94)$ \\
\hline$<65$ & $329(73.8)$ \\
\hline$\geq 65$ (including $\geq 75)$ & $117(26.2)$ \\
\hline$\geq 75$ & $32(7.2)$ \\
\hline Race $^{\mathrm{a}}$, Caucasian & $393(88.1)$ \\
\hline Body weight (kg), median (range) & $71.0(18-138)$ \\
\hline \multicolumn{2}{|l|}{$\begin{array}{l}\text { Renal impairment at initiation of } \\
\text { daptomycin }\end{array}$} \\
\hline $30<\mathrm{CrCl}<50 \mathrm{~mL} / \mathrm{min}$ & $42(9.4)$ \\
\hline $\mathrm{CrCl}<30 \mathrm{~mL} / \mathrm{min}$ & $25(5.6)$ \\
\hline Dialysis & $14(3.1)$ \\
\hline \multicolumn{2}{|l|}{ Underlying diseases ${ }^{\mathrm{b}}$ ( $>5 \%$ of patients) } \\
\hline Hematologic malignancy & $270(60.5)$ \\
\hline Immunosuppressed state & $177(39.7)$ \\
\hline Transplant & $124(27.8)$ \\
\hline $\begin{array}{l}\text { Anemia and/or all hematological } \\
\text { diseases }\end{array}$ & $90(20.2)$ \\
\hline Hypertension & $44(9.9)$ \\
\hline Cancer (solid organ) & $39(8.7)$ \\
\hline Diabetes mellitus & $36(8.1)$ \\
\hline Sepsis & $26(5.8)$ \\
\hline $\begin{array}{l}\text { Antibiotic therapy before initiation of } \\
\text { daptomycin }\end{array}$ & $319(71.5)$ \\
\hline Penicillins & $167(37.4)$ \\
\hline Glycopeptides & $145(32.5)$ \\
\hline Carbapenems & $98(22.0)$ \\
\hline \multicolumn{2}{|l|}{ Absolute neutrophil count $\left(\text { cells } / \mathrm{mm}^{3}\right)^{\mathrm{c}}$} \\
\hline$\leq 100$ & $223(50.0)$ \\
\hline $101-499$ & $77(17.3)$ \\
\hline $500-1000$ & $131(29.4)$ \\
\hline Missing data & $15(3.4)$ \\
\hline
\end{tabular}

Results are given as $n$ (\%) unless otherwise indicated $\mathrm{CrCl}$ creatinine clearance

${ }^{a}$ Missing data for $n=19$

b More than one underlying disease could be reported

${ }^{c}$ Lowest count at baseline or during daptomycin treatment 
Table 2 Type of primary infection

\begin{tabular}{|c|c|}
\hline Infection type $\mathrm{e}^{\mathrm{a}}$ & $\begin{array}{l}N=446 \\
n(\%)\end{array}$ \\
\hline Bacteremia & $188(42.2)$ \\
\hline Catheter related & $136(30.5)$ \\
\hline Non-catheter related & $52(11.7)$ \\
\hline Complicated skin and soft tissue infection & $62(13.9)$ \\
\hline Uncomplicated skin and soft tissue infections & $26(5.8)$ \\
\hline Foreign body and/or prosthetic infection & $20(4.5)$ \\
\hline Neutropenic fever & $19(4.3)$ \\
\hline Endocarditis & $15(3.4)$ \\
\hline $\begin{array}{l}\text { Osteomyelitis, non-prosthetic and prosthetic } \\
\text { device related }\end{array}$ & $11(2.5)$ \\
\hline Other $^{\mathrm{b}}$ & $105(23.5)$ \\
\hline
\end{tabular}

${ }^{a}$ A patient could have different infection types

b Includes surgical/non-surgical antibiotic prophylaxis, urinary tract infection/pyelonephritis, necrotizing infections, metastatic abscess, septic arthritis, central nervous system infection, and otherwise unspecified infections

The most common primary infections were catheter-related bacteremia (30.5\%; $n=136)$, non-catheter-related bacteremia (11.7\%; $n=52$ ), and complicated skin and soft tissue infection (cSSTI; 13.9\%; $n=62$ ) (Table 2).

\section{Microbiology}

Results of cultures were available for $83.0 \%$ $(n=370)$ of patients and were positive for $68.6 \%(n=254)$ of them (Table 3$)$. The most common pathogens were coagulase-negative staphylococci (CoNS), which were identified in $43.7 \% \quad(n=111)$ of patients with positive culture and $S$. aureus in $18.9 \%(n=48)$, with MRSA in $9.1 \%(n=23)$. VRE were reported in $5.5 \%(n=14)$ of patients.

\section{Previous and Concomitant Antibiotic Therapy}

Most patients $(71.5 \% ; n=319)$ received other antibiotic therapy before daptomycin treatment; the most frequent antibiotics were penicillins (37.4\%; $n=167)$, glycopeptides (32.5\%; $n=145)$, and carbapenems (22.0\%; $n=98)$ (Table 1).

When daptomycin was administered with concomitant antibiotics $(82.8 \% ; n=361)$, the most common antibiotics were carbapenems (51.2\%; $n=185)$, penicillins $(42.1 \% ; n=152)$, and aminoglycosides (19.9\%; $n=72)$.

\section{Daptomycin Prescribing Patterns}

Daptomycin was used empirically (i.e., before culture results were known) in 56.1\% $(n=250)$ of patients. Daptomycin was the first-line therapy for $27.5 \%(n=121)$ of patients and second-line therapy for $72.5 \%(n=319)$.

The most frequently prescribed doses of daptomycin were $6 \mathrm{mg} / \mathrm{kg} /$ day in $37.9 \%$ $(n=169)$ of patients and $4 \mathrm{mg} / \mathrm{kg} /$ day in $25.8 \% \quad(n=115)$ of patients. Although we cannot exclude that some patients received suboptimal daptomycin treatment, $4 \mathrm{mg} /$ $\mathrm{kg} /$ day is the approved dose of daptomycin for cSSTI without bacteremia. A total of $16.0 \%$ $(n=71)$ of patients received doses $>6$ and $\leq 10 \mathrm{mg} / \mathrm{kg} / \mathrm{day}$ and $16.0 \%(n=71)$ received doses $>4$ to $<6 \mathrm{mg} / \mathrm{kg} /$ day; $3.4 \%(n=15)$ of patients received $<4 \mathrm{mg} / \mathrm{kg} /$ day and $1.1 \%$ $(n=5)$ of patients had no record of dose.

The median duration of daptomycin therapy was 10.0 (range 1-58) days. 
Table 3 Microbiologic data in patients with neutropenia with positive cultures

\begin{tabular}{lc}
\hline Primary pathogens & $\begin{array}{l}\boldsymbol{N}=\mathbf{2 5 4} \\
\boldsymbol{n}(\%)\end{array}$ \\
\hline Coagulase-negative staphylococci (CoNS) & $111(43.7)$ \\
Staphylococcus epidermidis & $64(25.2)$ \\
Other & $47(18.5)$ \\
Staphylococcus aureus & $48(18.9)$ \\
Methicillin resistant (MRSA) & $23(9.1)$ \\
Methicillin susceptible (MSSA) & $20(7.9)$ \\
Methicillin susceptibility unknown & $5(2.0)$ \\
Enterococci (E. faecalis, E. faecium or other & $35(13.8)$ \\
species) & $23(9.1)$ \\
Enterococcus faecium & $9(3.5)$ \\
Enterococcus faecalis & $14(5.5)$ \\
Vancomycin resistant (E. faecalis and E. & \\
faecium) & $3(1.2)$ \\
Other species & $24(9.4)$ \\
Gram-negative bacilli & $8(3.1)$ \\
Viridans streptococci group & $28(11.0)$ \\
Other ${ }^{a}$ & \\
\hline
\end{tabular}

${ }^{a}$ Includes Gram-positive cocci, Staphylococcus species coagulase not specified, Streptococcus agalactiae or group B streptococci, Streptococcus dysgalactiae, Gram-negative cocci, Gram-positive bacilli, fungi/yeast, viruses, and invalid/ambiguous pathogen code

\section{Clinical Outcomes}

Clinical success (i.e., cured or improved) was reported for $77.1 \%(n=344)$ of patients $(46.2 \%$ were cured and $30.9 \%$ were improved). A total of $11.4 \%(n=51)$ of patients were considered as clinical failures and $11.4 \%(n=51)$ of patients were non-evaluable. Outcomes were comparable when analyzed by degree of neutropenia severity (Fig. 1). The success rate was $76.7 \%(n=171)$ for patients with severe neutropenia (ANC $\leq 100$ cells $/ \mathrm{mm}^{3}$ ), $80.5 \%$
( $n=62)$ for moderate neutropenia (101-499 cells $\left./ \mathrm{mm}^{3}\right)$, and $78.6 \% \quad(n=103)$ for mild neutropenia $\left(500-1000\right.$ cells $\left./ \mathrm{mm}^{3}\right)$. Clinical success was similar whether daptomycin was used as first-line or second-line therapy [79.3\% $(n=96)$ and $76.5 \% \quad(n=244)$, respectively]. Success rate by primary infection type ranged from $73.1 \%(n=19)$ for uncomplicated skin and soft tissue infections (uSSTI) to $93.3 \%$ ( $n=14$ ) for endocarditis (Fig. 2). The clinical success rates by infecting pathogen were high for CoNS $(85.6 \% ; n=95)$ and S. aureus $(77.1 \%$; $n=39$ ) (Fig. 3). The clinical success rates were similar regardless of daptomycin dose or dose range. Higher clinical success rates were observed with increased duration of daptomycin therapy (Fig. 4). The overall time to improvement was achieved within a median of 3 (range 1-30) days from initiation of daptomycin treatment.

\section{Safety}

AEs and SAEs, regardless of their relation to daptomycin, were reported in $19.3 \%(n=86)$ and $15.2 \% \quad(n=68)$ of patients, respectively (Table 4). Elevated serum creatine phosphokinase (CPK) was reported as an $\mathrm{AE}$ for one patient, but was considered to be unrelated to daptomycin by the investigator. CPK was measured at baseline for 216 patients and most (89.8\%) had normal values $(\leq 1 \times$ upper limit of normal; ULN). Three (1.4\%) patients had elevated CPK levels $(>10 \times$ ULN) at baseline. An elevation in CPK was observed in two patients (from $\leq 10 \times \mathrm{ULN}$ at baseline to $>10 \times$ ULN) during the study. There were no AEs of musculoskeletal and connective tissue disorders.

The total number of deaths was 50 (11.2\%) during the study and were all unrelated to the study drug. 


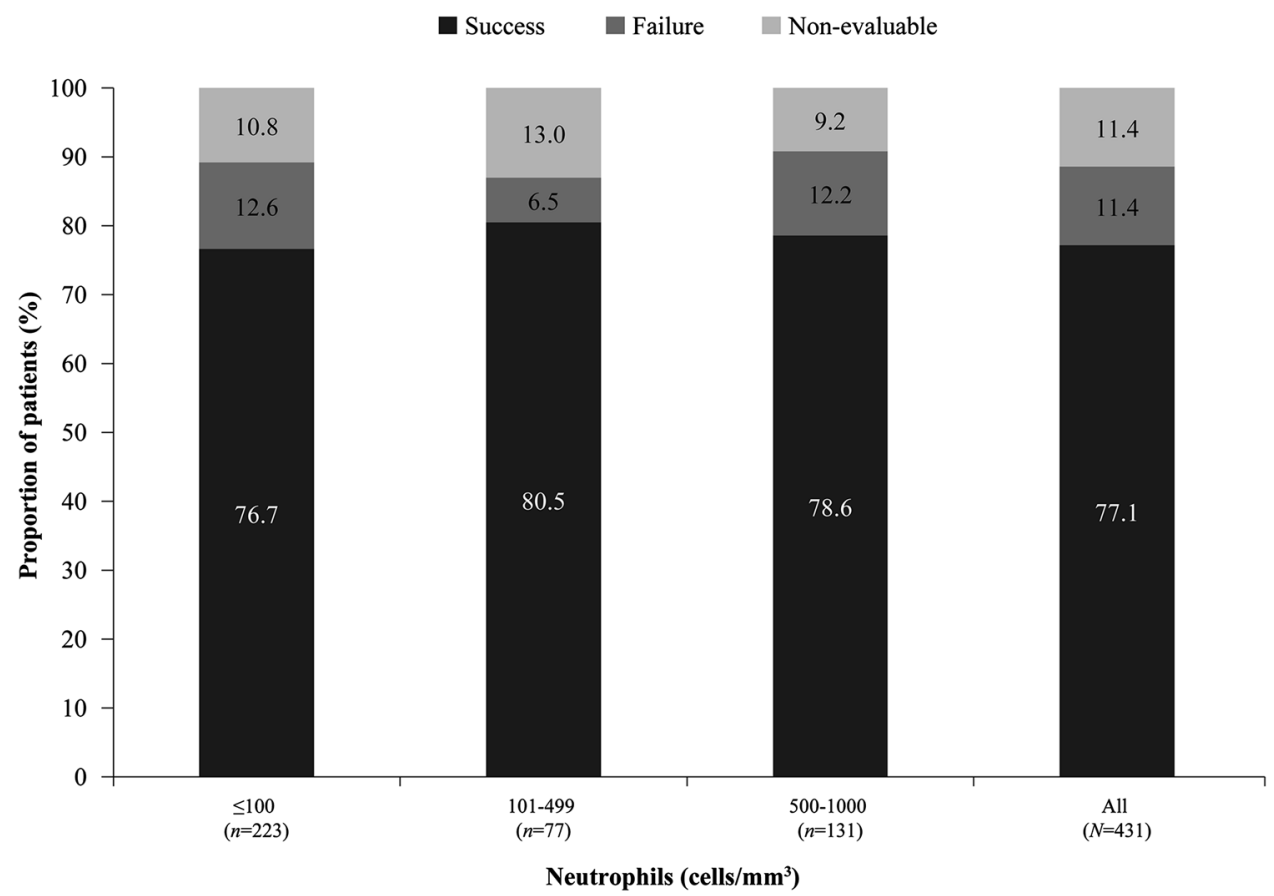

Fig. 1 Clinical outcome by degree of neutropenia severity. Neutropenia severity was missing for $n=15$ patients

\section{DISCUSSION}

Among the 446 patients with neutropenia from the EU-CORE registry who received daptomycin, there was a high clinical success rate $(77.1 \%)$. The success rates did not differ according to neutropenia severity, as previously reported in the CORE study [17].

Most study patients (56.1\%) received daptomycin as empirical treatment. Indeed, the main objective of initial empirical antibiotic therapy in patients with neutropenia is to prevent serious morbidity and mortality resulting from bacterial infections [5]. Empirical antibiotic regimen in febrile patients with neutropenia should be based on the risk status of the patient [2]. However, despite decades of well-performed clinical trials, no single empirical therapeutic regimen for the initial treatment of febrile patients with neutropenia has emerged as being clearly superior to others $[5,21]$. Thus, a recent Cochrane review concluded that the empirical routine addition of glycopeptides to anti-Gram-positive antibiotic treatment does not improve the outcomes of febrile patients with neutropenia and cancer [22].

Both the increasing incidence and the array of antibiotic-resistant pathogens have become important challenges in the treatment of patients with neutropenia [23, 24]. Thus, both vancomycin and linezolid have limitations with respect to their use in patients with neutropenia. First, vancomycin is no longer a standard recommendation in initial antibiotic therapy for fever and neutropenia [5]. In addition, a series of studies showed a relation between a higher vancomycin MIC of the infecting pathogen and a worse clinical outcome of patients with an MRSA infection [10]. Second, although linezolid produced similar outcomes in febrile patients with neutropenia compared with vancomycin [25], it is not bactericidal and might not be as 


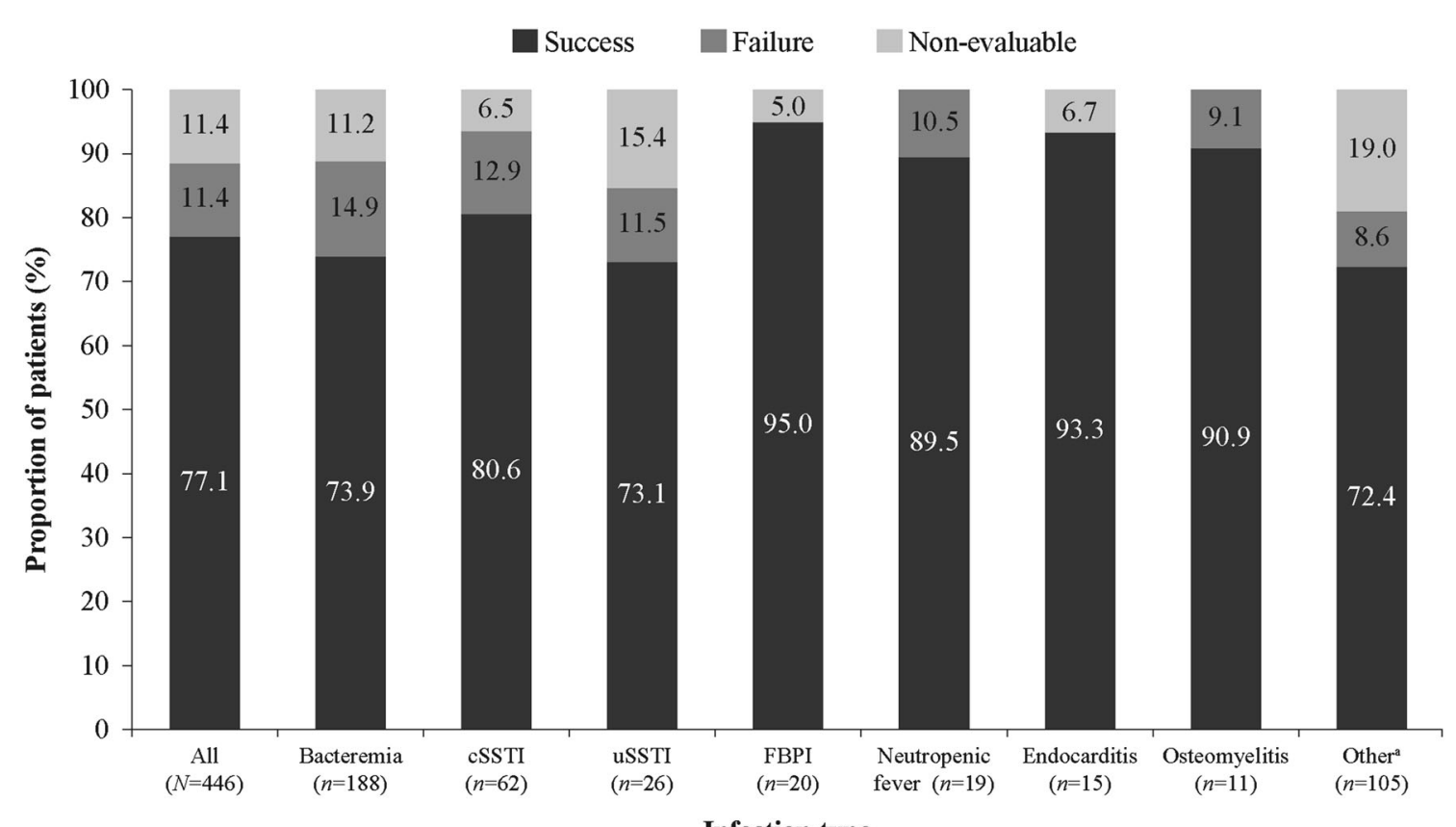

Infection type

Fig. 2 Clinical outcome by primary infection type. cSSTI complicated skin and soft tissue infection, FBPI foreign body/ prosthetic infection, $u S S T I$ uncomplicated skin and soft tissue infection. ${ }^{a}$ Includes surgical/non-surgical antibiotic prophylaxis, urinary tract infection/pyelonephritis, necrotizing infections, metastatic abscess, septic arthritis, central nervous system infection, and otherwise unspecified infections

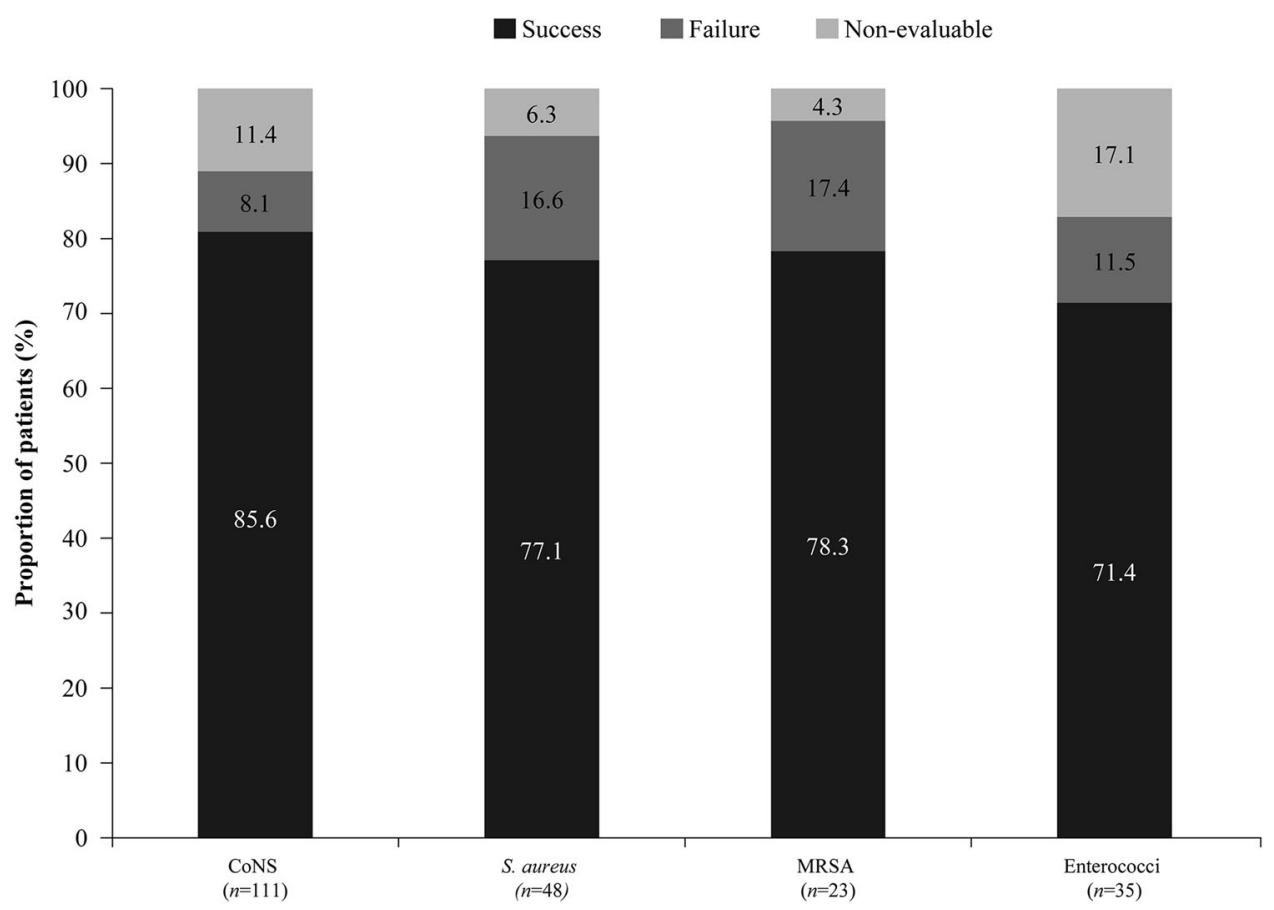

Fig. 3 Clinical outcome by selected primary pathogens. CoNS coagulase-negative staphylococci, $M R S A$ methicillin-resistant Staphylococcus aureus, enterococci included Enterococcus faecalis, Enterococcus faecium and other Enterococcus species 


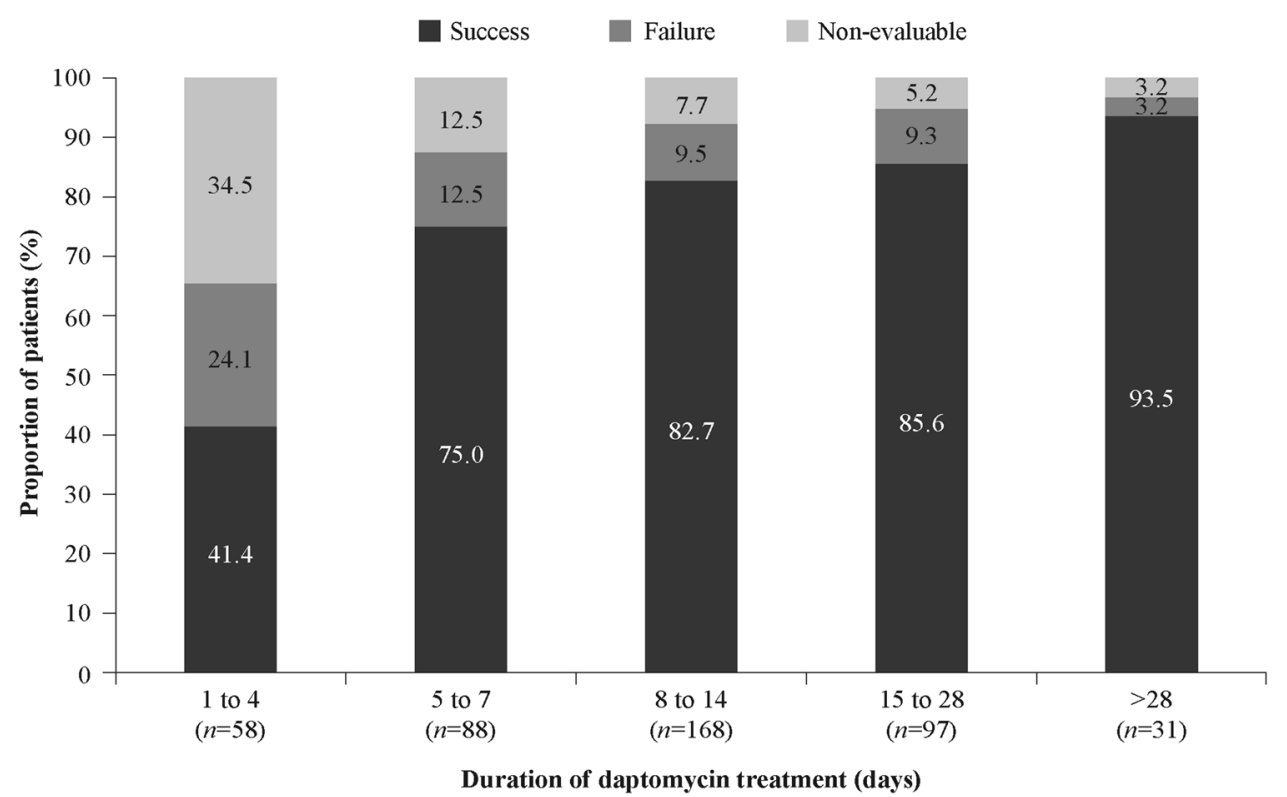

Fig. 4 Overall clinical outcome by duration of daptomycin therapy

effective as antibiotics such as daptomycin in eradicating catheter-related MRSA embedded in biofilm [26]. Finally, SAEs associated with linezolid were reported after its commercial release, including cases of lactic acidosis, peripheral and optic neuropathy, and serotonin syndrome [27].

Considering its once-daily administration, short infusion time, and good safety profile, daptomycin can be suggested as a therapeutic option of interest in patients with neutropenia, as reported in the Infectious Diseases Society of America (IDSA) guidelines [5]. This favorable profile of daptomycin was confirmed in our analysis. Thus, the high clinical success rate reported at the end of daptomycin therapy was obtained with a median time to improvement of 3 days. Of interest, the clinical success rates were comparable for first-line $(79.3 \%)$ or second-line $(76.5 \%)$ treatments. There are only a few reports on the use of daptomycin in the treatment of patients with neutropenia and Gram-positives infections. Poutsiaka et al. reported a clinical success rate in four out of nine (44\%) patients who had VRE bacteremia during episodes of fever and neutropenia [16]. Rolston et al. reported an overall clinical success rate as high as $90 \%$ in 84 patients with cancer and neutropenia [6]. There were some case reports on the use of daptomycin subsequent to vancomycin-induced neutropenia [15] or the use of a combination of daptomycin and gentamicin in a neutropenic patient [14]. The CORE study registry reported a high clinical success rate $(85 \%)$ in 186 patients with neutropenia and documented Gram-positive infections [17]. Daptomycin appears to be also suitable for outpatient therapy in cases of low-risk neutropenia [6].

In addition to its high effectiveness, our analysis showed that daptomycin was well tolerated. Only seven (1.6\%) patients experienced AEs possibly related to daptomycin and $27 \quad(6.1 \%)$ patients discontinued daptomycin therapy because of AEs. Before the optimization of the dosing 
Table 4 Safety of daptomycin treatment in patients with neutropenia

\begin{tabular}{lc}
\hline Safety parameters & $\begin{array}{l}\boldsymbol{N} \boldsymbol{n ( \% )} \\
n(46\end{array}$ \\
\hline Any AE & $86(19.3)$ \\
AEs possibly related to daptomycin & $7(1.6)$ \\
AEs leading to drug discontinuation & $27(6.1)$ \\
Any SAE & $68(15.2)$ \\
SAEs possibly related to daptomycin & $3(0.7)$ \\
SAEs leading to drug discontinuation & $24(5.4)$ \\
AEs occurring in $>1 \%$ patients & \\
Septic shock & $11(2.5)$ \\
Multiorgan failure & $9(2.0)$ \\
Neutropenia & $9(2.0)$ \\
Renal failure acute & $6(1.3)$ \\
Respiratory failure & $6(1.3)$ \\
Sepsis & $6(1.3)$ \\
\hline
\end{tabular}

$A E$ adverse event, $S A E$ serious $\mathrm{AE}$

interval with a once-daily regimen, reversible elevated serum CPK, and skeletal muscle toxicity had been reported [28]. In clinical trials with once-daily daptomycin, elevated CPK was reported in $7 \%$ of patients receiving $6 \mathrm{mg} / \mathrm{kg} /$ day, thus leading to study discontinuation in only $2.5 \%$ of patients. Elevated CPK was not reported in patients receiving $4 \mathrm{mg} / \mathrm{kg} /$ day $[29,30]$. Our safety data show that the impact of daptomycin was minimal on the serum CPK level because only one patient had elevated CPK during treatment (unrelated to daptomycin, according to the investigator) and there were no AEs related to musculoskeletal disorders. There were no new or unexpected safety findings.

There are some limitations in our analysis that are inherent to non-comparative, non-blinded, and retrospective studies. A central laboratory was not used in this registry and, therefore, microbiologic results and susceptibility to antibiotics could be different across the different centers. Moreover, the clinical outcomes were subjectively assessed by the local investigators. Most patients received concomitant antibiotic treatments and, thus, the success of treatment related to daptomycin remains uncertain. Nevertheless, our data have been recorded in real-world conditions and the criteria of selection of patients were not stringent in contrast to randomized clinical trials. A large number of institutions could participate without restrictions. The only selection criteria for these daptomycin-treated patients were neutropenia at baseline or during daptomycin therapy, and not participating in interventional studies with daptomycin. These selection criteria and the absence of restriction on concomitant or prior antibiotic treatments enabled the inclusion of various infections. Thus, patients who urgently needed treatment because of their conditions were included in the registry, but would have been excluded in a controlled clinical trial. Although the population was almost unselected, the overall clinical success rate was high, thus suggesting that daptomycin was effective in a real-world population of patients with neutropenia.

\section{CONCLUSION}

Real-world data from the EU-CORE registry study suggest that daptomycin is effective and well tolerated in patients with neutropenia and Gram-positive infections. Results are consistent with those observed in previous studies in patients with neutropenia and cancer.

\section{ACKNOWLEDGMENTS}

Sponsorship, article processing charges, and the open-access charge for this study were funded 
by Novartis Pharma AG. All named authors meet the International Committee of Medical Journal Editors (ICMJE) criteria for authorship of this manuscript, take responsibility for the integrity of the work as a whole, and have given final approval to the version to be published. Medical writing support was provided by Farid Khalfi (Novartis Ireland Ltd, Dublin, Ireland) and Francis Beauvais (independent medical writer). Funding for writing was provided by Novartis Pharma AG.

Conflict of interest. Felix Keil received honoraria from Novartis for speaking. George L. Daikos received research grants from Novartis. Athanasios Skoutelis received honoraria from Gilead, BMS, MSD, and Pfizer. Rashidkhan Pathan is an employee of Novartis Healthcare Pvt. Ltd. Kamal Hamed is an employee of Novartis Pharmaceuticals Corporation.

Compliance with ethics guidelines. The study has been performed in accordance with the ethical standards as laid down in the 1964 Declaration of Helsinki and its later amendments or comparable ethical standards. The protocol was approved by the health authority and the Institutional Review Board (IRB) or Ethics Committee (EC) in each country and written informed consent was obtained according to the requirements of the IRB or EC and/or the local data privacy regulations.

Open Access. This article is distributed under the terms of the Creative Commons Attribution-NonCommercial 4.0 International License (http://creativecommons.org/licenses/ by-nc/4.0/), which permits any noncommercial use, distribution, and reproduction in any medium, provided you give appropriate credit to the original author(s) and the source, provide a link to the Creative Commons license, and indicate if changes were made.

\section{REFERENCES}

1. Pene F, Percheron S, Lemiale V, et al. Temporal changes in management and outcome of septic shock in patients with malignancies in the intensive care unit. Crit Care Med. 2008;36:690-6.

2. de Naurois J, Novitzky-Basso I, Gill MJ, et al. Management of febrile neutropenia: ESMO clinical practice guidelines. Ann Oncol. 2010;21(Suppl $5): v 252-6$.

3. Villafuerte-Gutierrez $\mathrm{P}$, Villalon L, Losa JE, Henriquez-Camacho C. Treatment of febrile neutropenia and prophylaxis in hematologic malignancies: a critical review and update. Adv Hematol. 2014;2014:986938.

4. Legrand M, Max A, Schlemmer B, Azoulay E, Gachot B. The strategy of antibiotic use in critically ill neutropenic patients. Ann Intensive Care. 2011;1:22.

5. Freifeld AG, Bow EJ, Sepkowitz KA, et al. Clinical practice guideline for the use of antimicrobial agents in neutropenic patients with cancer: 2010 update by the Infectious Diseases Society of America. Clin Infect Dis. 2011;52:e56-93.

6. Rolston KV, McConnell SA, Brown J, Lamp KC. Daptomycin use in patients with cancer and neutropenia: data from a retrospective registry. Clin Adv Hematol Oncol. 2010;8(249-56):90.

7. Bubalo JS, Munar MY, Cherala G, Hayes-Lattin B, Maziarz R. Daptomycin pharmacokinetics in adult oncology patients with neutropenic fever. Antimicrob Agents Chemother. 2009;53:428-34.

8. Liu C, Bayer A, Cosgrove SE, et al. Clinical practice guidelines by the infectious diseases society of America for the treatment of methicillin-resistant Staphylococcus aureus infections in adults and children. Clin Infect Dis. 2011;52:e18-55.

9. Rybak MJ, Lomaestro BM, Rotschafer JC, et al. Vancomycin therapeutic guidelines: a summary of consensus recommendations from the Infectious Diseases Society of America, the American Society of Health-System Pharmacists, and the Society of Infectious Diseases Pharmacists. Clin Infect Dis. 2009;49:325-7.

10. Holland TL, Fowler VG Jr. Vancomycin minimum inhibitory concentration and outcome in patients 
with Staphylococcus aureus bacteremia: pearl or pellet? J Infect Dis. 2011;204:329-31.

11. Bionda N, Pitteloud JP, Cudic P. Cyclic lipodepsipeptides: a new class of antibacterial agents in the battle against resistant bacteria. Future Med Chem. 2013;5:1311-30.

12. Li Mortin, Li T, Van Praagh AD, et al. Rapid bactericidal activity of daptomycin against methicillin-resistant and methicillin-susceptible Staphylococcus aureus peritonitis in mice as measured with bioluminescent bacteria. Antimicrob Agents Chemother. 2007;51:1787-94.

13. Steenbergen JN, Alder J, Thorne GM, Tally FP. Daptomycin: a lipopeptide antibiotic for the treatment of serious Gram-positive infections. J Antimicrob Chemother. 2005;55:283-8.

14. Barber GR, Lauretta J, Saez R. A febrile neutropenic patient with Enterococcus gallinarum sepsis treated with daptomycin and gentamicin. Pharmacotherapy. 2007;27:927-32.

15. Mergenhagen KA, Pasko MT. Daptomycin use after vancomycin-induced neutropenia in a patient with left-sided endocarditis. Ann Pharmacother. 2007;41:1531-5.

16. Poutsiaka DD, Skiffington S, Miller KB, Hadley S, Snydman DR. Daptomycin in the treatment of vancomycin-resistant Enterococcus faecium bacteremia in neutropenic patients. $\mathrm{J}$ Infect. 2007;54:567-71.

17. Rolston KV, Besece D, Lamp KC, et al. Daptomycin use in neutropenic patients with documented Gram-positive infections. Support Care Cancer. 2014;22:7-14.

18. Gonzalez-Ruiz A, Beiras-Fernandez A, Lehmkuhl H, et al. Clinical experience with daptomycin in Europe: the first 2.5 years. J Antimicrob Chemother. 2011;66:912-9.

19. Flowers CR, Seidenfeld J, Bow EJ, et al. Antimicrobial prophylaxis and outpatient management of fever and neutropenia in adults treated for malignancy: American Society of Clinical Oncology clinical practice guideline. J Clin Oncol. 2013;31:794-810.

20. Dohmen PM, Guleri A, Capone A, et al. Daptomycin for the treatment of infective endocarditis: results from a European registry. J Antimicrob Chemother. 2013;68:936-42.
21. Antoniadou A, Giamarellou H. Fever of unknown origin in febrile leukopenia. Infect Dis Clin N Am. 2007;21:1055-90.

22. Paul M, Dickstein Y, Borok S, Vidal L, Leibovici L. Empirical antibiotics targeting Gram-positive bacteria for the treatment of febrile neutropenic patients with cancer. Cochrane Database Syst Rev. 2014;1:CD003914.

23. Alp S, Akova M. Management of febrile neutropenia in the era of bacterial resistance. Ther Adv Infect Dis. 2013;1:37-43.

24. Morris PG, Hassan T, McNamara $M$, et al. Emergence of MRSA in positive blood cultures from patients with febrile neutropenia-a cause for concern. Support Care Cancer. 2008;16:1085-8.

25. Jaksic B, Martinelli G, Perez-Oteyza J, et al. Efficacy and safety of linezolid compared with vancomycin in a randomized, double-blind study of febrile neutropenic patients with cancer. Clin Infect Dis. 2006;42:597-607.

26. Raad I, Hanna H, Jiang $\mathrm{Y}$, et al. Comparative activities of daptomycin, linezolid, and tigecycline against catheter-related methicillin-resistant Staphylococcus bacteremic isolates embedded in biofilm. Antimicrob Agents Chemother. 2007;51:1656-60.

27. Narita M, Tsuji BT, Yu VL. Linezolid-associated peripheral and optic neuropathy, lactic acidosis, and serotonin syndrome. Pharmacotherapy. 2007;27:1189-97.

28. Oleson FB Jr, Berman CL, Kirkpatrick JB, et al. Once-daily dosing in dogs optimizes daptomycin safety. Antimicrob Agents Chemother. 2000;44:2948-53.

29. Arbeit RD, Maki D, Tally FP, Campanaro E, Eisenstein BI. The safety and efficacy of daptomycin for the treatment of complicated skin and skin-structure infections. Clin Infect Dis. 2004;38:1673-81.

30. Fowler VG Jr, Boucher HW, Corey GR, et al. Daptomycin versus standard therapy for bacteremia and endocarditis caused by Staphylococcus aureus. N Engl J Med. 2006;355:653-65. 